\title{
Identification and Characterization of Pestalotioid Fungi Causing Leaf Spots on Mango in Southern China
}

\author{
Juan Shu, ${ }^{1,2}$ Zhihe Yu, ${ }^{1}$ Wenxiu Sun, ${ }^{1}$ Jiang Zhao, ${ }^{1}$ Qili Li, ${ }^{2, \dagger}$ Lihua Tang, ${ }^{2}$ Tangxun Guo, ${ }^{2}$ Suiping Huang, ${ }^{2}$ Jianyou Mo, ${ }^{2, \dagger}$ \\ Tom Hsiang, ${ }^{3}$ and Shuming $\mathrm{Luo}^{4}$ \\ ${ }^{1}$ College of Life Sciences, Yangtze University, Jingzhou, Hubei, 434025, China \\ ${ }^{2}$ Institute of Plant Protection, Guangxi Academy of Agricultural Sciences and Guangxi Key Laboratory of Biology for Crop Dis- \\ eases and Insect Pests, Nanning, Guangxi, 530007, China \\ ${ }^{3}$ School of Environmental Sciences, University of Guelph, Guelph, Ontario, Canada \\ ${ }^{4}$ Plant Breeding Institute, The University of Sydney, Cobbitty, NSW 2570, Australia
}

\begin{abstract}
Mango is an economically important fruit crop in southern China. However, leaf spots restrict the development of mango trees, reducing the yield and production. Pestalotioid fungi are one of the major agents causing leaf spots on mango. During 2016 and 2017, 21 isolates of pestalotioid fungi associated with leaf spots on mango leaves were collected from five provinces in southern China: Guangxi, Hainan, Yunnan, Guangdong, and Fujian. All 21 isolates were subjected to morphological characterization and DNA sequence analysis. The morphological data were combined with analyses of concatenated sequences of the ITS (internal transcribed spacer), TEF 1- $\alpha$ (translation

anacardiacearum, $P$. asiatica, $P$. photinicola, $P$. saprophyta, $P$. trachicarpicola, and Pseudopestalotiopsis ampullacea. Pathogenicity test results showed that all these species could cause symptoms. On detached mango leaves (cv. Tainong), early foliar symptoms on leaves were small yellow-to-brown lesions. Later, these spots expanded with uneven borders, turned white to gray, and coalesced to form larger gray patches. To our knowledge, this is the first description of $N$. clavispora, $P$. adusta, $P$. asiatica, $P$. photinicola, $P$. saprophyta, $P$. trachicarpicola, or Ps. ampullacea as causal agents for leaf spots on mango worldwide.
\end{abstract} elongation factor), and TUB2 ( $\beta$-tubulin) for higher resolution of the species identity of these isolates. The results showed that these isolates belong to Neopestalotiopsis clavispora, Pestalotiopsis adusta, P.
Keywords: mango, leaf spots, Neopestalotiopsis, Pestalotiopsis, Pseudopestalotiopsis, morphology, phylogeny
As a fruit-producing tree species in the Anacardiaceae, mango (Mangifera indica L.) is an important tropical and subtropical crop (Mukherjee 1953). In many countries, mango production is a pillar industry, and it is very important in China (Li et al. 2013; Nan et al. 2017). However, leaf spots restrict the development of mango trees, reducing the yield and production of mango, and pestalotioid fungi are one of the major agents causing leaf spots on mango (Okigbo and Osuinde 2003). Mango is cultivated mainly in southern provinces such as Guangxi, Hainan, Yunnan, Sichuan, Guangdong, Guizhou, and Fujian. In 2014, the total planted area of this fruit in mainland China was over 173,000 ha, and the production amounted to almost 1.5 million tons (Nan et al. 2017).

Leaf spots on mango are known to be caused by fungal species in genera such as Pestalotia, Botryodiplodia, Fusariella, and Macrophoma (Okigbo and Osuinde 2003). Recently, leaf spots have become increasingly more serious on mango seedlings, with incidence exceeding 20 to 30\% in China (Ding et al. 2010). Xiao et al. (1995) from 1985-1995 and Long et al. (2011) from 2008-2010 investigated and identified diseases of mango in China, and they found that leaf spots

${ }^{\dagger}$ Corresponding authors: Q. L. Li; 65615384@qq.com; liqili@gxaas.net and J. Y. Mo; mojianyou@gxaas.net

Funding: The research was supported by National Natural Science Foundation of China (Grant no. 31600029, 31560526), Guangxi Natural Science Foundation (Grant no. 2016GXNSFCB380004), Guangxi Key Laboratory of Biology for Crop Disease and Insect Pests (17-259-47-KF-1), and Foundation for Development of Science and Technology Guangxi Academy of Agricultural Sciences (2018YM22).

The author(s) declare no conflict of interest.

Accepted for publication 11 November 2019.

(C) 2020 The American Phytopathological Society are common and harmful. Mango leaf spots have also been reported in India, Indonesia, Philippines, France, South Africa, Brazil, and the United States. The incidence of leaf spots reached 70 to $100 \%$ per field in Italy (Ismail et al. 2013). The damage and loss caused by mango pathogens are unacceptable to fruit growers, retailers, and consumers (Johnson 1996).

Currently, there are more than 220 established pestalotioid species (CABI Bioscience database, 2005) found on various hosts worldwide. Among these 220 recognized pestalotioid species (CABI Bioscience database, 2005), there are at least 62 that are considered pathogenic (Ge et al. 2009), and among pathogenic species, 24 have been recorded as plant pathogens in Taiwan, China (Sawada 1944), and 38 as plant pathogens in Japan (Kishi 1988). However, only a few species in this genus have been reported on mango. For example, $P$. glandicola was the first reported causing mango leaf spot in India by Ullasa and Rawal (1985), while $P$. mangiferae was reported causing mango leaf spots in Taiwan, China (Ko et al. 2007). Other recent reports on new pestalotioid fungi associated with mango include: $P$. anacardiacearum in Yunnan, China, and $P$. samarangensis in Thailand (Maharachchikumbura et al. 2013a); as well as $P$. uvicola and $P$. clavispora in Italy (Ismail et al. 2013).

Leaf spots can lead to premature defoliation and eventual tree death (Qi 1994). However, there are limited reports to characterize and identify pathogens associated with mango. The genus Pestalotia was described as having fusiform conidia composed of six cells, and appendages in the apical and basal extremes (De Notaris 1839). Just over a hundred years later, the genus was divided into three genera, based on the number of cells comprising the conidia (Steyaert 1955). Specifically, conidia of the genera Pestalotia, Pestalotiopsis, and Truncatella possess 6, 5, and 4 cells, respectively (Steyaert 1955). Even after more than 60 years, this classification system is still in current use (Maharachchikumbura et al. 2011). However, there are controversies about generic divisions among pestalotioid fungi (Maharachchikumbura et al. 2014). Sutton (1980) used electron microscopy to examine the development of the cell wall for two species 
of pestalotioid and the species Pestalotia pezizoides, and the findings further supported Steyaert's classification (Griffiths and Swart 1974).

Until the 1990s, the taxonomy of pestalotioid and related genera was based on stable conidial characteristics such as the pigmentation of the three medium cells of the conidia, which is versicolorous in Neopestalotiopsis and concolorous in other pestalotioid species (Maharachchikumbura et al. 2011, 2012). However, using conidial characteristics for species identification for pestalotioid genera has been controversial due to the large variability of morphological characteristics such as colony color, texture, and shape and conidial characteristics when grown on culture media (Egger 1995; Hu et al. 2007). Recently, pestalotioid was redefined by analyzing the sequence of the $18 \mathrm{~S}$ together with the previous multiple-gene analyses by Maharachchikumbura et al. (2014), clearly showing three highly diverse lineages, and leading to the introduction of two new genera: Neopestalotiopsis and Pseudopestalotiopsis (Maharachchikumbura et al. 2014; Tsai et al. 2018). Maharachchikumbura et al. (2012) and Liu et al. (2017) evaluated 10 groups of primers for phylogenetic analyses and found that the internal transcribed spacer (ITS) region, the $\beta$-tubulin (TUB2), and the translation elongation factor (TEF1- $\alpha$ ) genes were optimal for resolving pestalotioid fungi.

The purpose of this study was to identify the species of pestalotioid fungi which caused mango leaf spots in several provinces of southern China using morphological observation, molecular identification, and pathogenicity testing.

\section{Materials and Methods}

Fungal isolation. During 2016 and 2017, mango leaves with leaf spots were collected from five major mango growing areas in China (Guangxi, Hainan, Yunnan, Guangdong, and Fujian) with at least one sample from each site (Fig. 1). Tissues were cut to $5 \times 5 \mathrm{~mm}$ pieces and soaked in $75 \%$ ethanol for $10 \mathrm{~s}$ and $5 \%$ hypochlorite for $1 \mathrm{~min}$, followed by three washes with autoclaved water for $3 \mathrm{~min}$. The samples were placed onto potato dextrose agar (PDA) and incubated at $28^{\circ} \mathrm{C}$ under $12 / 12 \mathrm{~h}$ light/darkness for 3-5 days. After colonies grew out, the hyphal margins were chosen for subculturing onto fresh PDA and incubated at $28^{\circ} \mathrm{C}$ for 14 to 21 days. Isolates were purified by single-spore transfers (Liu et al. 2003) and stored for further study. For isolate HN51 that did not produce conidia on regular PDA, sterilized pine needles were added to PDA to induce conidial production following Marques et al. (2013).

Morphological identification. Single-spore isolates were subcultured on PDA at $28^{\circ} \mathrm{C}$ and incubated up to 7 days, and then 5-mmdiameter plugs from colony margins were placed in the center of each 90-mm-diameter plate, with five replications per isolate. The color and culture diameters (two perpendicular directions) of each colony were recorded after 3,7 , and 14 days at $28^{\circ} \mathrm{C}$, and hyphal growth rates ( $\mathrm{mm} /$ day) calculated. Measurements were made of conidia produced on PDA after 2 to 3 weeks at $25^{\circ} \mathrm{C}$.

DNA extraction, PCR, and sequencing. The 21 isolates were cultured on PDA overlaid with cellophane strips and incubated at $28^{\circ} \mathrm{C}$ for 5-7 days. Fungi mycelia $(100 \mathrm{mg})$ were scraped from the surface. Genomic DNA was extracted using the DNAsecure Plant Kit [Tiangen Biotech (Beijing) Co., Ltd.] and diluted to a DNA concentration of $1 \mathrm{ng} / \mu \mathrm{l}$. Gel electrophoresis was used to quantify the DNA. The ITS region was amplified using primer pair ITS4/ITS5 (White et al. 1990), the TUB2 gene using BT2a/BT2b (Glass and Donaldson 1995; O'Donnell and Cigelnik 1997), and TEF1- $\alpha$ using EF1-526F/EF1-1567R (Rehner 2001).

PCR was done in a $25-\mu l$ reaction volume containing $11.5 \mu$ l of sterilized distilled water, $10.5 \mu \mathrm{l} 2 \times$ Ex Taq Master Mix (Shanghai Sangon Biotech Co., Ltd.), $2.0 \mu$ l of each $10 \mu \mathrm{M}$ primer $(\mathrm{R}+\mathrm{F})$, and $1 \mu \mathrm{l}$ template DNA. The PCR protocols were set as follows: an initial denaturing step of $3 \mathrm{~min}$ at $95^{\circ} \mathrm{C}$, followed by 35 cycles of denaturation at $95^{\circ} \mathrm{C}$ for $30 \mathrm{~s}$, annealing at $55^{\circ} \mathrm{C}$ for $30 \mathrm{~s}$ (for ITS), and elongation at $72^{\circ} \mathrm{C}$ for $30 \mathrm{~s}$, a final extension was performed at $72^{\circ} \mathrm{C}$ for $10 \mathrm{~min}$. The annealing temperatures for TEF1$\alpha$ and TUB2 were $58^{\circ} \mathrm{C}$ and $61^{\circ} \mathrm{C}$, respectively. The PCR products were sent to Nanning Guotuo Company for sequencing in both directions, and a consensus sequence generated of each amplified region. The sequences of the ITS region, and the TUB2 and TEF1- $\alpha$ genes for all 21 isolates were submitted to GenBank (Table 1).

Phylogenetic analyses. DNAMAN (version 5.2.2; Lynnon Biosoft) was used to edit and assemble sequences, and produce consensus sequences, which were identified by basic local alignment search
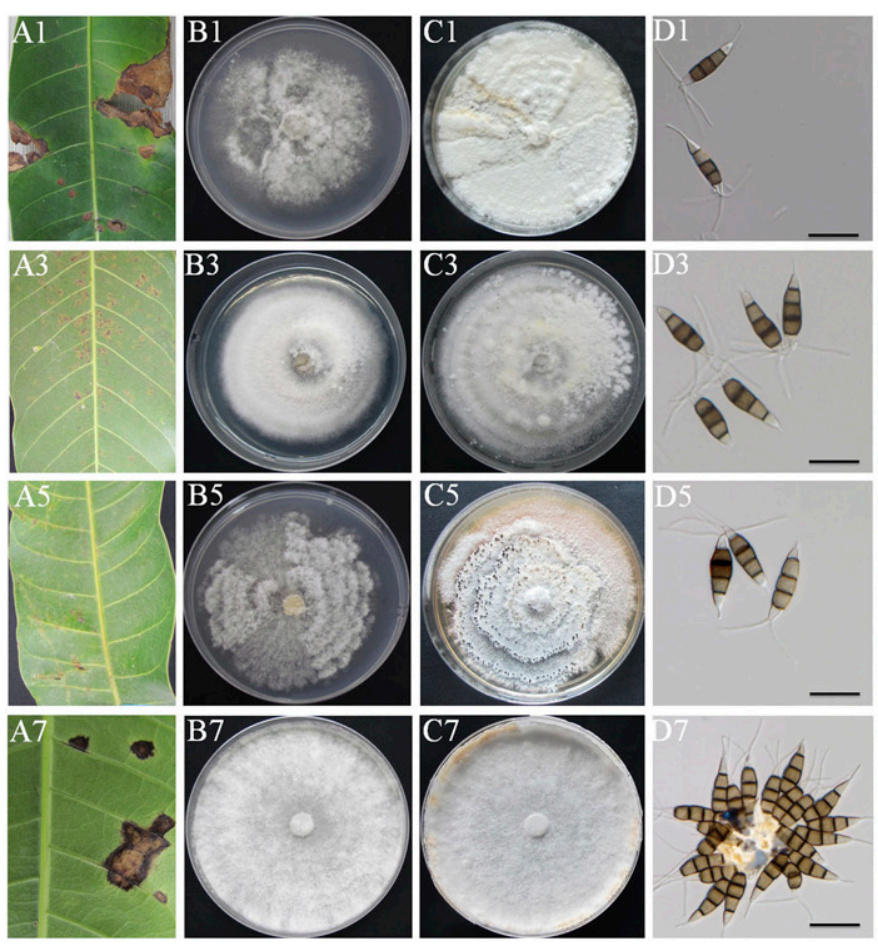
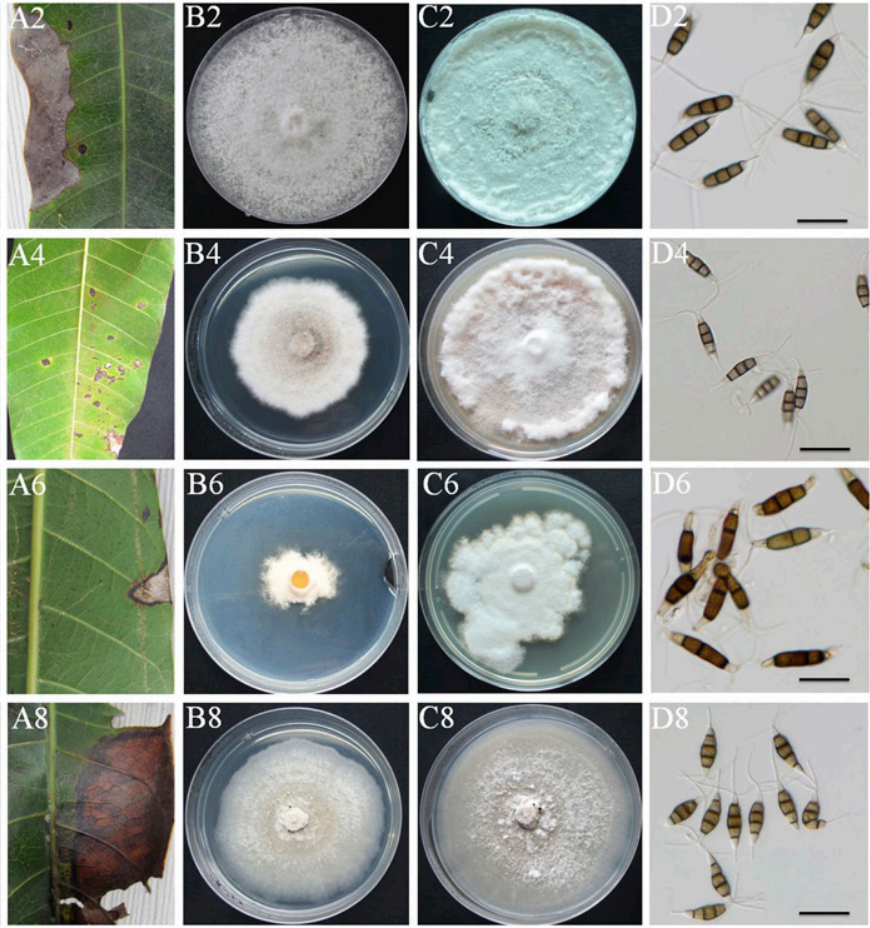

Fig. 1. A1 shows symptoms in the field, $\mathrm{B} 1$ has colony growth on $\mathrm{PDA}$ after 7 days at $28^{\circ} \mathrm{C}, \mathrm{C} 1$ has growth on $\mathrm{PDA}$ after 14 days at $28^{\circ} \mathrm{C}$, and $\mathrm{D} 1$ shows conidial features for Pestalotiopsis saprophyta; A2, B2, C2, and D2, Pseudopestalotiopsis ampullacea; A3, B3, C3, and D3, Neopestalotiopsis clavispora; A4, B4, C4, and D4, P. trachicarpicola; A5, B5, $\mathrm{C} 5$, and D5, P. asiatica; A6, B6, C6, and D6, P. anacardiacearum; A7, B7, C7, and D7, P. adusta; and A8, B8, C8, and D8, P. photinicola. 
tool (BLAST, Altschul et al. 1990) against the NCBI nucleotide database. Sequences from extype or ex-epitype isolates of pestalotioid fungi species from GenBank were also selected for phylogenetic analyses, including Seiridium camelliae as outgroup (Table 2). Sequence alignments for each locus and the combined loci were analyzed with ClustalX (v. 1.83) (Thompson et al. 1997). Where sequences were too short, the missing areas were filled in by consensus sequence. Phylogenetic trees were constructed with MEGA10 (version 10.0) using the Neighbor-Joining (NJ) and Maximum Likelihood (ML) method.

Pathogenicity tests on mango leaves. All 21 isolates were used for pathogenicity testing on detached mango leaves (cv. Tainong) in controlled conditions, with a randomized complete block design. The leaves were disinfected by immersion in $75 \%$ alcohol for $10 \mathrm{~s}$, then in $1 \% \mathrm{NaClO}$ for $1 \mathrm{~min}$, and finally rinsed three times in sterilized distilled water for $3 \mathrm{~min}$. The fresh young leaves (collected from
Tiandong, Guangxi province) were inoculated using the following procedure: multiple punctures were made with a sterilized toothpick in a 5-mm-diameter circle, followed by inoculation with a 5-mmdiameter mycelial plug from a 7-day-old PDA culture, and PDA plugs were mock-inoculated onto mango leaves as controls. Leaves were then placed in transparent plastic boxes which were first washed with $75 \%$ alcohol and lined with autoclaved filter paper moistened with sterilized distilled water to maintain high humidity. For each isolate, there were 10 replicate leaves. The boxes were then sealed and incubated at $28^{\circ} \mathrm{C}$ under $12 / 12 \mathrm{~h}$ of light/darkness for 5 days. Virulence was assessed by measuring lesion length at 5 days postinoculation (DPI) in two perpendicular directions on each leaf. The data were checked for normality and then subjected to analysis of variance (ANOVA). The model was lesion length as a function of fungal isolate. Mean comparisons involved post hoc analysis by LSD $(P=0.05)$ using Data Processing System software (DPS 3.0,

Table 1. Pestalotioid isolates collected from mango leaves in southern China, with species, geographic origin, and GenBank accession details

\begin{tabular}{|c|c|c|c|c|c|}
\hline \multirow[b]{2}{*}{ Isolate } & \multirow[b]{2}{*}{ Species } & \multirow[b]{2}{*}{ Geographic area } & \multicolumn{3}{|c|}{ GenBank accession number ${ }^{\mathbf{z}}$} \\
\hline & & & ITS & TEF1- $\alpha$ & TUB2 \\
\hline FJ2-G-2 & Neopestalotiopsis clavispora & Xiamen, Fujian & MK228984 & MK512478 & MK360925 \\
\hline HN40-1 & N. clavispora & Jiangchang, Hainan & MK228985 & MK512479 & MK360926 \\
\hline HN51 & N. clavispora & Dongyun, Hainan & MK228986 & MK512480 & MK360927 \\
\hline YN27-2-2 & N. clavispora & Ganzuang, Yuanjiang & MK228987 & MK512481 & MK360928 \\
\hline YB27-3 & Pestalotiopsis adusta & Yuanjiang, Yunnan & MK228988 & MK512482 & MK360929 \\
\hline YB56-1 & P. adusta & Xishuangbanna, Yunnan & MK228989 & MK512483 & MK360930 \\
\hline FY10-12 & P. anacardiacearum & Pumei, Fujian & MK228990 & MK512484 & MK360931 \\
\hline HN37-4 & P. anacardiacearum & Jiangchang, Hainan & MK228991 & MK512485 & MK360932 \\
\hline YB41-2 & P. anacardiacearum & Yuanyang, Yunnan & MK228992 & MK512486 & MK360933 \\
\hline YN30-1 & P. asiatica & Ganzuang, Yuanjiang & MK228993 & MK512487 & MK360934 \\
\hline HN44-1 & P. asiatica & Jiangchang, Hainan & MK228994 & MK512488 & MK360935 \\
\hline YN54-2 & P. asiatica & Xishuangbanna, Yunnan & MK228995 & MK512489 & MK360936 \\
\hline FY7-3 & P. photinicola & Nanshan, Fujian & MK228996 & MK512490 & MK360937 \\
\hline YB28-2 & P. photinicola & Yuanjiang, Yunnan & MK228997 & MK512491 & MK360938 \\
\hline GD22-1 & P. saprophyta & Leizhou, Guangdong & MK228998 & MK512492 & MK360939 \\
\hline YN44-2-1 & P. saprophyta & Xishuangbanna, Yunnan & MK228999 & MK512493 & MK360940 \\
\hline HN56-2 & P. trachicarpicola & Sanya, Hainan & MK229000 & MK512494 & MK360941 \\
\hline GX17-1 & Pseudopestalotiopsis ampullacea & Tianyang, Guangxi & MK229001 & MK512495 & MK360942 \\
\hline GX23-1 & Ps. ampullacea & Tiandong, Guangxi & MK229002 & MK512496 & MK360943 \\
\hline HN41-1 & Ps. ampullacea & Jiangchang, Hainan & MK229003 & MK512497 & MK360944 \\
\hline YB36-2 & Ps. ampullacea & Honghe, Yunnan & MK229004 & MK512498 & MK360945 \\
\hline
\end{tabular}

${ }^{\mathrm{z}}$ ITS $=$ internal transcribed spacer; TEF1- $\alpha=$ translation elongation factor; TUB2 $=\beta$-tubulin.

Table 2. Sequences from pestalotioid fungi obtained from GenBank and used as reference in this study

\begin{tabular}{|c|c|c|c|c|c|c|}
\hline \multirow[b]{2}{*}{ Species } & \multirow[b]{2}{*}{ Isolate } & \multirow[b]{2}{*}{ Host } & \multirow[b]{2}{*}{ Location } & \multicolumn{3}{|c|}{ GenBank accession $^{z}$} \\
\hline & & & & ITS & TUB2 & TEF1- $\alpha$ \\
\hline Neopestalotiopsis clavispora & MFLUCC $12-0281$ & Magnolia sp. & China & JX398979 & JX399014 & JX399045 \\
\hline N. clavispora & MFLUCC12-0280 & Magnolia sp. & China & JX398978 & JX399013 & JX399044 \\
\hline Pestalotiopsis adusta & ICMP 6088 & On refrigerator door & Fiji & JX399006 & JX399037 & JX399070 \\
\hline P. adusta & MFLUCC10-146 & Syzygium sp. & Thailand & JX399007 & JX399038 & JX399071 \\
\hline P. anacardiacearum & IFRDCC 2397 & Mangifera indica & China & KC247154 & KC247155 & KC247156 \\
\hline P. asiatica & MFLUCC 12-0286 & Unidentified tree & China & JX398983 & JX399018 & JX399049 \\
\hline P. camellia & MFLUCC12-0277 & Camellia japonica & China & JX399010 & JX399041 & JX399074 \\
\hline P. clavata & MFLUCC12-0268 & Buxus sp. & China & JX398990 & JX399025 & JX399056 \\
\hline P. diversiseta & MFLUCC12-0287 & Rhododendron sp. & China & JX399009 & JX399040 & JX399073 \\
\hline P. furcata & MFLUCC12-0054 & Camellia sinensis & Thailand & JQ683724 & JQ683708 & JQ683740 \\
\hline P. inflexa & MFLUCC12-0270 & Unidentified tree & China & JX399008 & JX399039 & JX399072 \\
\hline P. intermedia & MFLUCC12-0259 & Unidentified tree & China & JX398993 & JX399028 & JX399059 \\
\hline P. linearis & MFLUCC12-0271 & Trachelospermum sp. & China & JX398992 & JX399027 & JX399058 \\
\hline P. photinicola & GZCC $16-0028$ & Punica granatum & China & KY092404 & KY047663 & KY047662 \\
\hline P. saprophyta & MFLUCC 12-0282 & Magnolia sp. & China & JX398982 & JX399017 & JX399048 \\
\hline P. trachicarpicola & OP068 & Trachycarpus fortunei & China & JQ845947 & JQ845945 & JQ845946 \\
\hline P. unicolor & MFLUCC12-0275 & Unidentified tree & China & JX398998 & JX399029 & JX399063 \\
\hline Pseudopestalotiopsis ampullacea & LC6618 & Camellia japonica & China & KX895025 & KX895358 & KX895244 \\
\hline Seiridium camelliae & SD096 & Camellia sinensis & China & JQ683725 & JQ683709 & JQ683741 \\
\hline
\end{tabular}

${ }^{\mathrm{z}}$ ITS $=$ internal transcribed spacer; TEF1- $\alpha=$ translation elongation factor; TUB2 $=\beta$-tubulin. 
Tang and Zhang 2013). The lesion diameters were compared with mycelial growth using linear regression analysis.

\section{Results}

Fungal isolation. Representative samples of leaf spots on mango were collected to represent different stages of the disease from different provinces in China (Guangxi, 34; Hainan, 58; Yunnan, 56; Guangdong, 35; and Fujian, 36). Early foliar symptoms on leaves were small brown spots with haloes (Fig. 1 plates A3, A4, and A5); when the disease spots continued to expand, the color gradually deepened to reddish-brown (Fig. 1 plates A1 and A8) or black-brown (Fig. 1 plate A7), finally becoming gray (Fig. 1 plates A2 and A6). Over 400 isolates were obtained from 219 samples, and the ITS was sequenced for each of these. Among these, 21 pestalotioid isolates were retained, and the TUB2 and TEF1- $\alpha$ genes were sequenced. As well, colony characteristics (color and shape), appendages, and conidial morphology (color, size, and shape) were observed (Table 3). The 21 isolates included six from Hainan, nine from Yunnan, one from Guangdong, two from Guangxi, and three from Fujian province. Other genera were identified by ITS in this research, such as Colletotrichum, Alternaria, Phomopsis, and Fusarium (Table 4), and they have been retained for analysis in subsequent research.

Morphological and cultural characterization. After 7 days at $28^{\circ} \mathrm{C}$, the colonies on PDA grew up to 7 to $9 \mathrm{~cm}$ in diameter with wavy edges, off-white color, dense aerial growth, and a reverseside yellowish color. After 2 to 3 weeks, black fruiting bodies were present (Fig. 1). There were considerable variations in mycelial growth rate (mean value) among 21 isolates in this study (Table 3). We also compared the mycelial growth rates by isolate (Fig. 2A).

All species had five-celled conidia, but showed some differences in conidial morphology: $P$. saprophyta conidia had transparent end cells and the middle three cells were brown, dark or light (Fig. 1, D1); Ps. ampullacea conidia had transparent end cells, and the middle three cells were dark brown (Fig. 1, D2); N. clavispora conidia had end cells transparent or grayish, and the middle three cells were brown or light brown (Fig. 1, D3); P. trachicarpicola conidia had end cells transparent or grayish, and the middle three cells were brown (Fig. 1, D4); P. asiatica conidia had transparent end cells, and the middle three cells were dark brown or light brown (Fig. 1, D5); $P$. anacardiacearum conidia had end cells brown or beige, and the middle three cells were russet (Fig. 1, D6); P. adusta conidia had beige end cells, and the middle three cells were brown (Fig. 1, D7); and $P$. photinicola conidia had transparent or gray end cells, and the middle three cells were hazel (Fig. 1, D8). The average conidial sizes for $P$. saprophyta were $23.6 \pm 0.7 \mu \mathrm{m} \times 6.4 \pm 0.3 \mu \mathrm{m} ; P$ s. ampullaceal, $24.9 \pm 1.3 \mu \mathrm{m} \times 6.4 \pm 0.2 \mu \mathrm{m} ; N$. clavispora, $23.5 \pm$ $1.9 \mu \mathrm{m} \times 6.4 \pm 0.3 \mu \mathrm{m} ; P$. trachicarpicola, $18.4 \pm 0.0 \mu \mathrm{m} \times 4.9 \pm$ $0.0 \mu \mathrm{m} ; P$. asiaticae, $19.7 \pm 2.9 \mu \mathrm{m} \times 6.5 \pm 0.3 \mu \mathrm{m} ; P$. anacardiacearum, $24.7 \pm 1.9 \mu \mathrm{m} \times 7.6 \pm 0.5 \mu \mathrm{m} ; P$. adusta, $20.1 \pm 1.4 \mu \mathrm{m} \times 5.8 \pm$ $1.4 \mu \mathrm{m}$; and $P$. photinicola, $19.6 \pm 1.0 \mu \mathrm{m} \times 6.0 \pm 0.5 \mu \mathrm{m}$. There were significant differences between conidial size and growth rates among the isolates (Table 3). Each species had two or three appendages. The

Table 4. Preliminary identification of 471 fungal isolates from 219 mango leaf samples from five provinces in China

\begin{tabular}{lrrrrr}
\hline & \multicolumn{5}{c}{ Isolate number from different provinces of China } \\
\cline { 2 - 6 } Genus $^{\mathbf{z}}$ & Yunnan & Hainan & Fujian & Guangdong & Guangxi \\
\hline Colletotrichum & 34 & 23 & 44 & 16 & 65 \\
Lasiodiplodia & 14 & 5 & 1 & 3 & 6 \\
Botryosphaeria & 2 & 4 & 10 & 8 & 10 \\
Neofusicoccum & 11 & 2 & 14 & 3 & 3 \\
Neopestalotiopsis & 3 & 3 & 2 & 0 & 0 \\
Pseudopestalotiopsis & 1 & 1 & 0 & 0 & 4 \\
Pestalotiopsis & 15 & 4 & 7 & 1 & 0 \\
Daldinia & 10 & 1 & 14 & 0 & 10 \\
Phomopsis & 6 & 5 & 18 & 0 & 3 \\
Fusarium & 12 & 2 & 3 & 1 & 3 \\
Phyllosticta & 0 & 1 & 0 & 0 & 14 \\
Alternaria & 1 & 8 & 0 & 0 & 3 \\
Pseudoplagiostoma & 0 & 0 & 0 & 0 & 5 \\
Others & 8 & 9 & 9 & 2 & 4 \\
\hline
\end{tabular}

${ }^{\mathrm{z}}$ The fungal isolates were identified based on morphology and ITS sequences.

Table 3. Morphology represented by conidial size, mycelial growth rate, and pathogenicity denoted by lesion diameter on detached mango leaves of pestalotioid isolates collected in this study

\begin{tabular}{|c|c|c|c|c|c|}
\hline Species & Isolate & Conidial size $(\mu \mathrm{m})^{\mathrm{w}}$ & Growth rate $(\mathbf{m m} / \mathbf{d})^{x}$ & Lesion diameter $(\mathbf{m m})^{\mathbf{y}}$ & Appendage length $(\mu \mathrm{m})^{\mathrm{z}}$ \\
\hline Neopestalotiopsis clavispora & FJ2-G-2 & $19.4 \pm 0.2 \times 6.0 \pm 0.1$ & $9.0 \pm 2.5 \mathrm{a}$ & $7.8 \pm 0.1 \mathrm{~b}$ & $17.87 \pm 0.7$ fghij \\
\hline N. clavispora & HN40-1 & $22.4 \pm 0.2 \times 5.8 \pm 0.1$ & $5.6 \pm 0.6$ bcde & $26.4 \pm 2.4 \mathrm{a}$ & $17.68 \pm 0.5$ fghij \\
\hline N. clavispora & HN51 & $23.6 \pm 0.3 \times 7.0 \pm 0.1$ & $5.6 \pm 0.5$ bcde & $13.3 \pm 0.1 \mathrm{ab}$ & $29.04 \pm 1.0 \mathrm{a}$ \\
\hline N. clavispora & YN27-2-2 & $28.5 \pm 0.3 \times 6.9 \pm 0.1$ & $6.1 \pm 0.3 \mathrm{bcde}$ & $11.7 \pm 0.1 \mathrm{~b}$ & $23.65 \pm 0.8$ ef \\
\hline Pestalotiopsis adusta & YB27-3 & $18.7 \pm 0.8 \times 7.2 \pm 0.3$ & $4.7 \pm 0.2$ ef & $7.0 \pm 0.0 \mathrm{~b}$ & $14.64 \pm 0.6 \mathrm{hij}$ \\
\hline P. adusta & YB56-1 & $21.4 \pm 0.2 \times 4.5 \pm 0.1$ & $6.6 \pm 0.7 \mathrm{bc}$ & $4.6 \pm 0.0 \mathrm{~b}$ & $13.5 \pm 0.7 \mathrm{ij}$ \\
\hline P. anacardiacearum & FY10-12 & $25.1 \pm 0.3 \times 7.2 \pm 0.1$ & $2.9 \pm 0.3 \mathrm{~g}$ & $5.7 \pm 0.1 \mathrm{~b}$ & $25.48 \pm 1.5 \mathrm{def}$ \\
\hline P. anacardiacearum & HN37-4 & $21.3 \pm 0.3 \times 7.1 \pm 0.1$ & $2.7 \pm 0.2 \mathrm{~g}$ & $2.6 \pm 0.1 \mathrm{~b}$ & $17.58 \pm 0.6$ fghij \\
\hline P. anacardiacearum & YB41-2 & $27.7 \pm 0.3 \times 8.5 \pm 0.1$ & $2.7 \pm 0.3 \mathrm{~g}$ & $4.1 \pm 0.0 \mathrm{~b}$ & $22.98 \pm 1.0 \mathrm{hij}$ \\
\hline P. asiatica & YN30-1 & $25.2 \pm 0.2 \times 6.1 \pm 0.1$ & $6.5 \pm 0.5 \mathrm{bcd}$ & $2.4 \pm 0.0 \mathrm{~b}$ & $19.16 \pm 1.1 \mathrm{fgh}$ \\
\hline P. asiatica & HN44-1 & $15.3 \pm 0.3 \times 6.3 \pm 0.1$ & $4.8 \pm 0.5 \mathrm{de}$ & $8.0 \pm 0.0 \mathrm{~b}$ & $12.69 \pm 0.5 \mathrm{j}$ \\
\hline P. asiatica & YN54-2 & $18.7 \pm 0.3 \times 7.1 \pm 0.1$ & $6.7 \pm 0.5 b c$ & $4.1 \pm 0.0 \mathrm{~b}$ & $18.75 \pm 0.7 \mathrm{fgh}$ \\
\hline P. photinicola & FY7-3 & $20.6 \pm 0.2 \times 5.5 \pm 0.1$ & $6.3 \pm 0.5$ bcde & $8.1 \pm 0.0 \mathrm{~b}$ & $16.35 \pm 0.5 \mathrm{hij}$ \\
\hline P. photinicola & YB28-2 & $18.6 \pm 0.2 \times 6.4 \pm 0.1$ & $3.2 \pm 0.2 \mathrm{fg}$ & $4.5 \pm 0.0 \mathrm{~b}$ & $17.2 \pm 0.8$ fghij \\
\hline P. saprophyta & GD22-1 & $22.9 \pm 0.2 \times 6.0 \pm 0.1$ & $5.5 \pm 0.4$ bcde & $10.0 \pm 0.0 \mathrm{~b}$ & $26.23 \pm 0.8 \mathrm{de}$ \\
\hline P. saprophyta & YN44-2-1 & $24.3 \pm 0.2 \times 6.3 \pm 0.1$ & $6.5 \pm 0.5 b c$ & $9.4 \pm 0.1 \mathrm{~b}$ & $25.39 \pm 0.8 \mathrm{~b}$ \\
\hline P. trachicarpicola & HN56-2 & $18.4 \pm 0.2 \times 4.9 \pm 0.1$ & $6.0 \pm 0.6$ bcde & $3.4 \pm 0.2 b$ & $18.39 \pm 0.6$ fghi \\
\hline Pseudopestalotiopsis ampullacea & GX17-1 & $25.8 \pm 0.2 \times 6.5 \pm 0.1$ & $7.1 \pm 0.5 b$ & $4.2 \pm 0.0 \mathrm{~b}$ & $24.63 \pm 0.9 \mathrm{def}$ \\
\hline Ps. ampullacea & GX23-1 & $21.5 \pm 0.2 \times 5.8 \pm 0.3$ & $7.0 \pm 0.5 \mathrm{~b}$ & $11.4 \pm 0.1 \mathrm{~b}$ & $12.33 \pm 1.7 \mathrm{c}$ \\
\hline Ps. ampullacea & HN41-1 & $27.9 \pm 0.7 \times 6.5 \pm 0.3$ & $2.9 \pm 0.4 \mathrm{~g}$ & $10.3 \pm 0.1 \mathrm{~b}$ & $23.69 \pm 0.9 \mathrm{ef}$ \\
\hline Ps. ampullacea & YB36-2 & $24.5 \pm 0.4 \times 6.6 \pm 0.1$ & $5.3 \pm 0.6 \mathrm{cde}$ & $15.6 \pm 0.1 \mathrm{ab}$ & $29.88 \pm 1.0 \mathrm{~d}$ \\
\hline
\end{tabular}

${ }^{w}$ The length and width of 100 conidia per isolate on PDA were measured after $14-21$ days at $28^{\circ} \mathrm{C}$.

$\mathrm{x}$ The colony diameter $(\mathrm{mm})$ was measured in two perpendicular directions. The colony diameter data were used to calculate the mycelial growth rate (mm/day). Means followed by a letter in common are not significantly different based on the test of least significant difference $(P=0.05)$.

y The virulence of each isolate was evaluated by measuring lesion length at 5 days postinoculation in two perpendicular directions on detached leaves.

$\mathrm{z}$ The length of appendages per isolate on PDA were measured after $14-21$ days at $28^{\circ} \mathrm{C}$. 
longest lengths (mean value) were found with $P$. anacardiacearum, 11-50 $\mu \mathrm{m}$, and the shortest were with P. adusta, 10-20 $\mu \mathrm{m}$.

Phylogenetic analysis. The sequences of ITS, TUB2, TEF1- $\alpha$ for all the isolates in this study were compared with sequences in GenBank and matches with high similarity ( $\geq 99 \%)$ were selected. Combining ITS, TUB2, and TEF1- $\alpha$ sequence data gave a total length of 2,019 bp, with 605 variable sites in this multigene alignment. Missing data were replaced by consensus sequence for $P s$. ampulla $(459 \mathrm{bp})$, P. photinicola $(44 \mathrm{bp})$, P. trachicarpicola (9 bp), and $P$. diversiseta $(9 \mathrm{bp})$. In this study, we used Seiridium camelliae as outgroup, and used NJ and ML methods to analyze the alignments. All species separated into three major clades (Fig. 3). The phylogenetic analysis revealed these species associations for isolates obtained in this study: $P$. anacardiacearum (FY10-12, YB41-2, and HN37-4), P. adusta (YB27-3 and YB56-1), P. asiatica (HN44-1, YN54-2, and YN30-1), P. photinicola (YB28-2 and FY7-3), P. trachicarpicola (HN56-2), P. saprophyta (GD22-1 and YN44-2-1), $N$. clavispora (HN51, HN40-1, YN27-2-2, and FJ2-G-2), and Ps. ampullacea (GX17-1, HN41-1, and YB36-2). Bootstrap support for these groupings ranged from 72 to 100 .

Pathogenicity on mango leaves. All 21 isolates produced symptoms on detached mango leaves, while the mock-inoculated PDA plugs did not. The data were normally distributed. The virulence (lesion sizes) for these isolates showed significant differences. The mean lesion lengths for $P$. saprophyta were $9.7 \pm 0.3 \mathrm{~mm}$; Ps. ampullacea, $10.4 \pm 2.4 \mathrm{~mm} ; N$. clavispora, $14.8 \pm 4.0 \mathrm{~mm} ; P$. trachicarpicola, $3.4 \pm 0.0 \mathrm{~mm} ; P$. asiatica, $4.8 \pm 1.7 \mathrm{~mm} ; P$. anacardiacearum, $4.1 \pm 0.9 \mathrm{~mm} ;$ P. adusta, $5.8 \pm 1.2 \mathrm{~mm}$; and P. photinicola, $6.3 \pm$ $1.8 \mathrm{~mm}$. The two largest lesion diameters (mean value) were $26.4 \mathrm{~mm}$ for $N$. clavispora, HN40-1 from Hainan, and $15.6 \mathrm{~mm}$ for Ps. ampullacea, YB36-2 from Yunnan. The two smallest lesion diameters (mean value) were $2.4 \mathrm{~mm}$ for $P$. asiatica, YN30-1 from Yunnan, and $2.6 \mathrm{~mm}$ for $P$. asiatica, HN37-4 from Hainan. There were no obvious statistically significant relationships observed between the virulence level and the fungi species tested, nor between virulence and origin. Correlation analysis did not reveal a statistically significant relationship between lesion diameter and mycelial growth for each species $(\mathrm{r}=0.5008, P=0.55)$ (Fig. 2B).

\section{Discussion}

Based on molecular and morphological characterization along with virulence assays, we found that 21 pestalotioid isolates from five provinces in China from mango leaf spots involve a variety of species from the genera Pestalotiopsis, Neopestalotiopsis, and Pseudopestalotiopsis. Except for $P$. anacardiacearum, the other seven fungi species including P. adusta, P. asiatica, P. saprophyta, P. trachicarpicola, $P$. photinicola, $N$. clavispora, and $P$ s. ampullacea are the first reports on mango in China.

Ismail et al. (2013) found 70 to $100 \%$ leaf spot incidence on mango in Italy during 2009 to 2010 . This incidence of leaf spots reduced the yield and production of mango, and can lead to unacceptable levels of loss for fruit growers (Johnson 1996). For other plants, previous research has reported that pestalotioid fungi can significantly damage hosts, such as loquat, oil palm, louts, and southern magnolia, with reported yield losses of $40 \%$ for oil palm in Columbia and South America (Ge et al. 2009).

Maharachchikumbura et al. (2012) reported 14 new species of Pestalotiopsis on various plants in China, but none of these were on mango. In this study, we found six species of Pestalotiopsis, including 13 isolates from Fujian (2), Hainan (4), Yunnan (6), and Guangdong (1). Neopestalotiopsis clavispora has been found on a variety of shrubby plants (Chamorro et al. 2016; Chen et al. 2016; Feng et al. 2014; González et al. 2012). In this study, we detected four isolates of this species from three provinces in China: Fujian (1), Hainan (2), and Yunnan (1). The genus Pseudopestalotiopsis has seven species (Joshi et al. 2009; Maharachchikumbura et al. 2016; Nozawa et al. 2017). In this study, we found a Ps. ampullacea from three provinces in China: Yunnan (1), Guangxi (2), and Hainan (1). These three genera mentioned above have not been previously reported on mango.

Traditionally, only morphological data has been available, and it was difficult to identify isolates to a species level, especially for isolates from Neopestalotiopsis, Pestalotiopsis, and Pseudopestalotiopsis that showed variability in their morphological characteristics (Keith et al. 2006). In this study, colony characteristics of the single-spore isolates were not stable when subcultured because the isolates varied in one or more traits. This phenomenon was previously reported by $\mathrm{Hu}$ et al. (2007) that the cultural characteristics (e.g., color, growth rate, and texture) of the colony for pestalotioid were flexible upon subculturing (Jeewon et al. 2003; Maharachchikumbura et al. 2011; Tejesvi et al. 2007). On the other hand, conidial characteristics were relatively stable, especially the length, width, and color of the conidia. These characteristics were more effective for resolving to genus, but not always dependable for resolving to species (Maharachchikumbura et al. 2011).

In 2014, two species, $N$. honoluluana and $N$. zimbabwana, were defined based on only six nucleotide differences among ITS, TUB, TEF1- $\alpha$, ACT, GAPDH, and LSU sequence (Maharachchikumbura et al. 2014). In contrast, Maharachchikumbura et al. (2012) found one $N$. foedans isolate with six nucleotide differences in the same genomic regions was still considered to be the same species as two other $N$. foedans isolates evaluated. In this study, we compared three genes (ITS, TUB2, TEF1- $\alpha$ ), and found that analysis of the concatenated sequences gave the finest resolution.

This research revealed the species diversity within the genera Pestalotiopsis, Neopestalotiopsis, and Pseudopestalotiopsis associated with mango leaf spots in China. Our study may accelerate the development of future epidemiological research for leaf spots on mango and other crops caused by pestalotioid fungi. Identification of the pathogen could facilitate selection of management strategies, such as appropriate chemistries and crop rotation, and the evaluation of resistance in mango germplasm to the different Neopestalotiopsis,
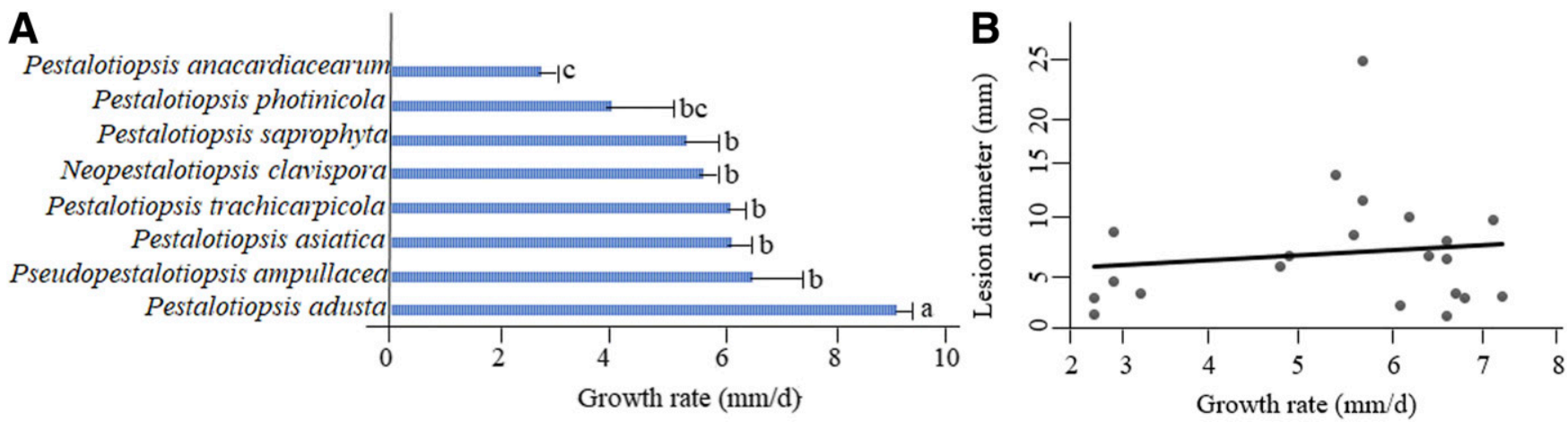

Fig. 2. A, Bar graph of mean growth rate per species based on 7 days of growth on $P D A$ at $28^{\circ} \mathrm{C}$; and $\mathbf{B}$, scatterplot showing relationship between lesion diameter at 5 dpi and mycelial growth rate of each species. $(Y=0.5008 X+5.6625, P=0.55)$. Mean bar sizes followed by a letter in common are not significantly different based on the test of Least Significant Difference $(P=0.05)$. 
Pseudopestalotiopsis, and Pestalotiopsis isolates. This finding of the presence of different species means that more studies are needed on whether these species differ in their sensitivity to different control agents and can lead to more focused studies on specific species, especially if their disease cycles do not entirely overlap. The discovery of pathogenicity differences between isolates from among different species can facilitate research of pathogenicity mechanisms and disease resistance breeding. It can also provide ideas for studies on leaf spots of other crops. In future studies, a greater number of samples should be examined. As well, genomic and transcriptomic analyses can reveal deeper insights into different species of pestalotioid fungi. The different species and different isolates may show varied sensitivity to different control agents, so this is also worthy of further study. Disease resistance breeding and plant biosecurity could be considered as well as other research on disease prevention and management.

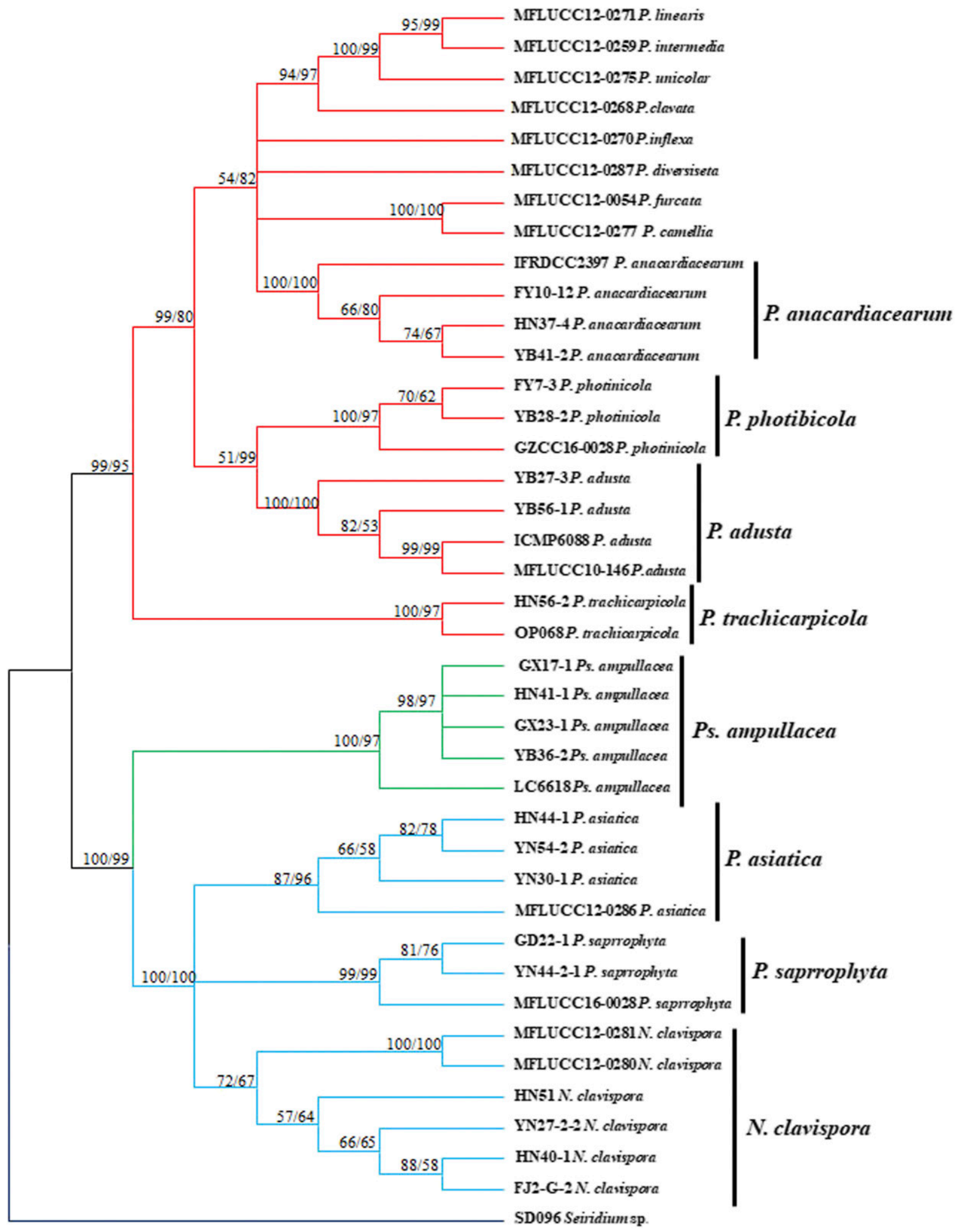

Fig. 3. Phylogenetic tree based on combined ITS, TUB2, and TEF1- $\alpha$ genes for 21 pestalotioid isolates from mango, plus 18 ex-type or ex-epitype pestalotioid isolates from GenBank. Seiridium camelliae was used as outgroup. Bootstrap values $>50 \%$ are given at the nodes (NJ/ML). 


\section{Literature Cited}

Altschul, S. F., Gish, W., Miller, W., Myers, E. W., and Lipman, D. J. 1990. Basic local alignment search tool. J. Mol. Biol. 215:403-410.

Chamorro, M., Aguado, A., and De los Santos, B. 2016. First report of root and crown rot caused by Pestalotiopsis clavispora (Neopestalotiopsis clavispora) on strawberry in Spain. Plant Dis. 100:1495.

Chen, Y., Zhang, A. F., Yang, X., Gu, C. Y., Kyaw, E. P., Yi, X. K., and Xu, Y. L. 2016. First report of Pestalotiopsis clavispora causing twig blight on highbush blueberry (Vaccinium corymbosum) in Anhui province of China. Plant Dis. 100:859.

De Notaris, G. 1839. Micromycetes italiei Dec II. Mere R Acad. Sci. Torino II. 3:80-81.

Ding, R., Wang, Y. L., Li, B. X., and Xie, C. P. 2010. Identification and characterization of mango leaf blight disease pathogen Pestalotiopsis mangiferae. Fruit Trees South. China 39:20-24.

Egger, K. N. 1995. Molecular analysis of ectomycorrhizal fungal communities. Can. J. Bot. 73, S1, Suppl. 1:1415-1422.

Feng, Y. R., Liu, B. S., and Sun, B. B. 2014. First report of leaf blotch caused by Pestalotiopsis clavispora on Rosa chinensis in China. Plant Dis. 98:1009.

Ge, Q. X., Chen, Y. X., and Xu, T. 2009. Flora fungorum sinicorum. Vol. 38, Pestalotiopsis. Science Press, Beijing.

Glass, N. L., and Donaldson, G. C. 1995. Development of primer sets designed for use with the PCR to amplify conserved genes from filamentous ascomycetes. Appl. Environ. Microbiol. 61:1323-1330.

González, P., Alaniz, S., Montelongo, M. J., Rauduviniche, L., Rebellato, J., Silvera-Pérez, E., and Mondino, P. 2012. First report of Pestalotiopsis clavispora causing dieback on blueberry in Uruguay. Plant Dis. 96:914.

Griffiths, D. A., and Swart, H. J. 1974. Conidial structure in two species of Pestalotiopsis. Trans. Br. Mycol. Soc. 62:295-304.

Hu, H., Jeewon, R., Zhou, D., Zhou, T., and Hyde, K. D. 2007. Phylogenetic diversity of endophytic Pestalotiopsis species in Pinus armandii and Ribes spp.: Evidence from rDNA and $\beta$-tubulin gene phylogenies. Fungal Divers. 24:1-22.

Ismail, A. M., Cirvilleri, G., and Polizzi, G. 2013. Characterisation and pathogenicity of Pestalotiopsis uvicola and Pestalotiopsis clavispora causing grey leaf spot of mango (Mangifera indica L.) in Italy. Eur. J. Plant Pathol. 135:619-625.

Jeewon, R., Liew, E. C., Simpson, J. A., Hodgkiss, I. J., and Hyde, K. D. 2003. Phylogenetic significance of morphological characters in the taxonomy of Pestalotiopsis species. Mol. Phylogenet. Evol. 27:372-383.

Johnson, G. I. 1996. Mango disease losses: Balancing economy and ecology. In V International Mango Symposium, 455, 575-586.

Joshi, S. D., Sanjay, R., Baby, U. I., and Mandal, A. K. A. 2009. Molecular characterization of Pestalotiopsis spp. associated with tea (Camellia sinensis) in southern India using RAPD and ISSR markers. Indian J. Biotechnol. 8:377-383.

Keith, L. M., Velasquez, M. E., and Zee, F. T. 2006. Identification and characterization of Pestalotiopsis spp. causing scab disease of guava, Psidium guajava, in Hawaii. Plant Dis. 90:16-23.

Ko, Y., Yao, K. S., Chen, C. Y., and Lin, C. H. 2007. First report of gray leaf spot of mango (Mangifera indica) caused by Pestalotiopsis mangiferae in Taiwan. Plant Dis. 91:1684.

Kishi, K. (ed.). 1988. Plant Disease in Japan. Zenkoku Noson Kyoiku Kyokai Co. Ltd. Tokyo, Japan.

Li, R., Huang, G., Su, M., Zhou, J., and Chen, Y. 2013. Status and developmental strategies of mango industry in China. J. S. Agric. 44:875-878.

Liu, A. R., Zhang, R. Y., and Xie, X. C. 2003. Isolation and identification of pathogen of banana crown rot. J. S. China U. Trop. Agric. 9:1-5.

Liu, F., Hou, L., Raza, M., and Cai, L. 2017. Pestalotiopsis and allied genera from Camellia, with description of 11 new species from China. Sci. Rep. 7:866-885.

Long, Y. Q., Wang, W. D., and Wang, M. C. 2011. Investigation of mango pests and diseases in Yunnan. Trop. Crop J. 32:508-513.

Maharachchikumbura, S. S., Guo, L. D., Cai, L., Chukeatirote, E., Wu, W. P., Sun, X., Crous, P. W., Bhat, D. J., McKenzie, E. H. C., Bahkali, A. H., and Hyde, K. D. 2012. A multi-locus backbone tree for Pestalotiopsis, with a polyphasic characterization of 14 new species. Fungal Divers. 56:95-129.

Maharachchikumbura, S. S., Guo, L. D., Chukeatirote, E., Bahkali, A. H., and Hyde, K. D. 2011. Pestalotiopsis-Morphology, phylogeny, biochemistry and diversity. Fungal Divers. 50:167-187.
Maharachchikumbura, S. S., Guo, L. D., Chukeatirote, E., McKenzie, E. H., and Hyde, K. D. 2013a. A destructive new disease of Syzygium samarangense in Thailand caused by the new species Pestalotiopsis samarangensis. Trop. Plant Pathol. 38:227-235.

Maharachchikumbura, S. S., Guo, L. D., Liu, Z. Y., and Hyde, K. D. 2016. Pseudopestalotiopsis ignota and Ps. camelliae spp. nov. associated with grey blight disease of tea in China. Mycol. Prog. 15:22.

Maharachchikumbura, S. S., Hyde, K. D., Groenewald, J. Z., Xu, J., and Crous, P. W. 2014. Pestalotiopsis revisited. Stud. Mycol. 79:121-186.

Maharachchikumbura, S. S., Zhang, Y., Wang, Y., and Hyde, K. D. 2013b. Pestalotiopsis anacardiacearum sp. nov. (Amphisphaeriaceae) has an intricate relationship with Penicillaria jocosatrix, the mango tip borer. Phytotaxa 99:49-57.

Marques, M. W., Lima, N. B., de Morais, M. A., Michereff, S. J., Phillips, A. J., and Câmara, M. P. 2013. Botryosphaeria, Neofusicoccum, Neoscytalidium and Pseudofusicoccum species associated with mango in Brazil. Fungal Divers. 61:195-208.

Mukherjee, S. K. 1953. The mango-Its botany, cultivation, uses and future improvement, especially as observed in India. Econ. Bot. 7:130-162.

Nan, N., Fu, Z. J., and Xu, J. C. 2017. Mango industrialized development in China. J. Y. Agric. U. 11:80-84.

Nozawa, S., Yamaguchi, K., Van Hop, D., Phay, N., Ando, K., and Watanabe, K. 2017. Identification of two new species and a sexual morph from the genus Pseudopestalotiopsis. Mycoscience 58:328-337.

O’Donnell, K., and Cigelnik, E. 1997. Two divergent intragenomic rDNA ITS2 types within a monophyletic lineage of the fungusfusariumare nonorthologous. Mol. Phylogenet. Evol. 7:103-116.

Okigbo, R. N., and Osuinde, M. I. 2003. Fungal leaf spot diseases of mango (Mangifera indica L.) in southeastern Nigeria and biological control with Bacillus subtilis. Plant Prot. Sci. 39:70-77.

Qi, P. k. 1994:Pages 1-275 in: Fungi Disease of Cultivated Plants in Guangdong Province. Guangdong Science and Technology Press, Guangzhou.

Rehner, S. 2001. Primers for Elongation Factor 1-a (EF1-a). Assembling the Fungal Tree of Life, Corvallis, OR. https://web.archive.org/web/20030827095957/ http://ocid.nacse.org/research/deephyphae/EF1primer.pdf.

Sawada, K. 1994. Investigation report on fungi from Taiwan. Vol. 1-11, Notes on the species of Pestalotia. Taiwan Governor's Office Agricultural Laboratory, Taiwan.

Solarte, F., Muñoz, C. G., Maharachchikumbura, S. S., and Álvarez, E. 2018. Diversity of Neopestalotiopsis and Pestalotiopsis spp., causal agents of Guava scab in Colombia. Plant Dis. 102:49-59.

Steyaert, R. L. 1955. Pestalotia, Pestalotiopsis et Truncatella. Bull. van den Rijksplantentuin, Brussels. Pages 191-199.

Sutton, B. C. 1980. The Coelomycetes: Fungi imperfecti with pycnidia, acervuli and stomata. Commonw. Mycol. I., England.

Tang, Q. Y., and Zhang, C. X. 2013. Data Processing System (DPS) software with experimental design, statistical analysis and data mining developed for use in entomological research. Insect Sci. 20:254-260.

Tejesvi, M. V., Kini, K. R., Prakash, H. S., Subbiah, V., and Shetty, H. S. 2007. Genetic diversity and antifungal activity of species of Pestalotiopsis isolated as endophytes from medicinal plants. Fungal Divers. 24:37-54.

Thompson, J. D., Gibson, T. J., Plewniak, F., Jeanmougin, F., and Higgins, D. G. 1997. The CLUSTAL_X windows interface: Flexible strategies for multiple sequence alignment aided by quality analysis tools. Nucleic Acids Res. 25: 4876-4882.

Tsai, I., Maharachchikumbura, S. S., Hyde, K. D., and Ariyawansa, H. A. 2018. Molecular phylogeny, morphology and pathogenicity of Pseudopestalotiopsis species on Ixora in Taiwan. Mycol. Prog. 17:941-952.

Ullasa, B. A., and Rawal, R. D. 1985. Occurrence of a new post-harvest disease of mango due to Pestalotiopsis glandicola. Acta Hortic.: 540-543.

White, T. J., Bruns, T., Lee, S. J. W. T., and Taylor, J. 1990. Amplification and direct sequencing of fungal ribosomal RNA genes for phylogenetics. Pages 315-322 in: PCR Protocols: A Guide to Methods and Applications. Academic Press, New York.

Xiao, Q. C., Yu, Z. T., and Zheng, J. H. 1995. Identification the species of mango diseases and pathogens. Trop. Crop J. 16:77-83. 